\title{
Microwaves in Textile Finishing, Yes or No
}

\section{Drago Katović}

University of Zagreb, Faculty of Textile Technology, Croatia

\section{Introduction}

According to well known physical definition, electromagnetic waves are oscillating electric and magnetic fields traveling together through space. In the electromagnetic radiation spectrum, shown in Figure 1. Microwaves $(300 \mathrm{MHz}-300 \mathrm{GHz})$ lie between radio wave $(\mathrm{RF})$ and infrared (IR) frequencies with a relatively large wavelength $(1 \mathrm{~m}-1 \mathrm{~mm})$. The term "microwave" was used for the first time in 1932, and its first usage was during the Second World War in radio communication and radar technology.

Electromagnetic waves are composed of two components: an electric wave or an electric field and a magnetic wave or magnetic field. The fields are in dynamic motion, and these two waves are in phase moving at 90 degrees towards each other. The orientation of this "package" can vary in the space through which it moves, which leads to what we call polarization, but the electric and magnetic components always maintain the right angle relationship to each other. Electromagnetic wave is formed by the electrical charge in the conductor that produces an electrical field in the spreading direction. The electrical field produces the magnetic field. The so-formed magnetic field reproduces the electrical field in the space. A microwave electromagnetic field, oscillating at $2.45 \mathrm{GHz}$, is the preferred frequency for heating applications. Microwave radiation is specially tuned to

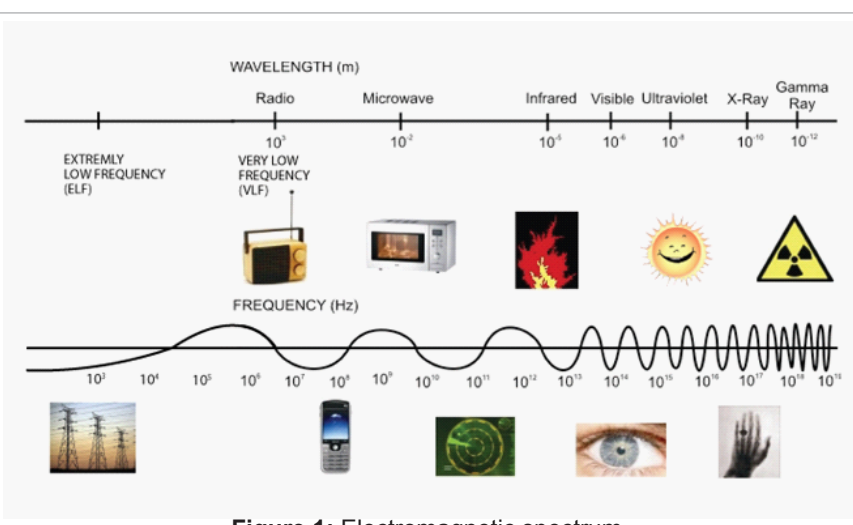

Figure 1: Electromagnetic spectrum.

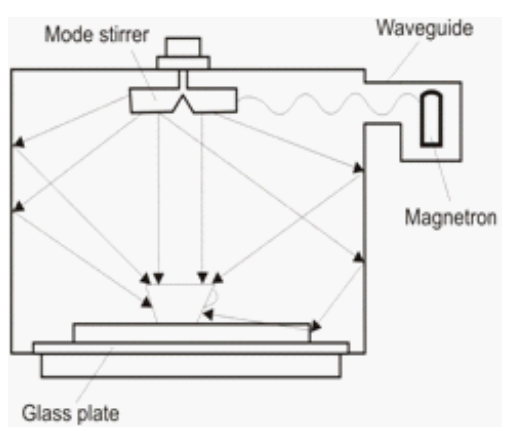

Figure 2: Microwave oven natural frequency of water molecules to maximize interactions.

The energy of the microwave photons is very low $(0,125 \mathrm{~kJ} / \mathrm{mol})$ relative to the typical energies for chemical bonds $(335-84 \mathrm{~kJ} / \mathrm{mol})$; thus microwave will not directly affect the molecular structure. They cannot change the electronic structure around atoms or among them, but they can interact with the electronic differences between atoms. High intensity microwaves, as in a microwave oven where they pass back and forth through the food millions of times, heat the material by producing molecular rotations and torsions. Until now, microwaves and RF have been used in agricultural and food processing industries to control insect growth in wheat, flour, stored grain and cereal products and to eradicate wood boring insects in timber. Insects are not likely to develop resistance to radiation as they often do to chemical insecticides. Microwaves have been used for food preparation, chemical sludge, medical waste organic synthesis, in analysis and curing of hi-tech polymers. Today they are widely accepted and spread to mobile phones, television, wireless commuter networks and some special applications such as rocket engines.

Chemical reactions can be accelerated due to selective absorption of electromagnetic energy by polar molecules, while non-polar molecules are inert to the electromagnetic radiation. Besides influencing dipole water molecules, an alternating electromagnetic field also acts on partially polar molecules of textiles such as polyurethane (PU), polyacrylonitrile (PAN), or polyamide (PA). Some important applications of microwaves come from their interaction with various types of material. The interaction of microwaves with dielectric materials causes net polarization of the substance. The overall net polarization creates a dipole moment. Dipole rotation is an interaction in which polar molecules or species try to align themselves with the rapidly changing electric field of the applied radiation. The motion of the molecule as it tries to orient to the field results in transfer of energy. The second way to transfer energy is ionic conduction that occurs if there are free ions or ionic species present in the heated substance heated.

Radio frequency (RF) and microwaves (MW) are forms of electromagnetic energy but differ in operating frequency and wavelength. Both are allocated specific bands of operation by international governments. Radio frequency dryers are operating

*Corresponding author: Dr.Drago Katović, Faculty of TextileTechnology, University of Zagreb, Croatia, Tel: +385 (01) 4877352; Fax: +385 (01) 4877352; E-mail: Drago.Katovic@ttf.hr

Received December 14, 2011; Accepted December 16, 2011; Published December 19, 2011

Citation: Katović D (2011) Microwaves in Textile Finishing, Yes or No. J Textile Sci Engg 1:e102. doi:10.4172/2165-8064.1000e102

Copyright: (c) 2011 Katović D. This is an open-access article distributed under the terms of the Creative Commons Attribution License, which permits unrestricted use, distribution, and reproduction in any medium, provided the original author and source are credited. 
with power from 10 till $100 \mathrm{~kW}$. Generally speaking, the efficiency of power utilization is far lower in a RF generator than a microwave unit, although the initial capital cost per KW of power output is higher. Selection of RF or microwave heating will depend on the product's physical properties and required process conditions for a particular application. Where penetration depth in excess of $15 \mathrm{~cm}$ is required and control of uniformity of heating is not a major issue, radio frequency offers a good solution. However, where there is uniformity of drying and moisture control is essential. For planar applications requiring belt widths in excess of $100 \mathrm{~cm}$, where edge-to-edge uniformity is essential, control of microwave energy is superior to RF.

\section{Radio frequency dryers}

Electromagnetic waves have been used in the textile industry finishing for the purpose of drying of thick materials, performed at radio frequency $(\mathrm{RF})$ dryers, operating at different frequencies between 10 and $30 \mathrm{MHz}$ with wavelengths of 30 to 10 meters. In these machines, energy is transferred by means of two metal electrodes plates, between which the fabric is transported on a conveyer belt. An alternating electric field is created between the electrodes, with alternating voltage created by on RF generator.

Under the influence of the alternating electric field, dipole water molecules start vibrating, which causes them to heat up
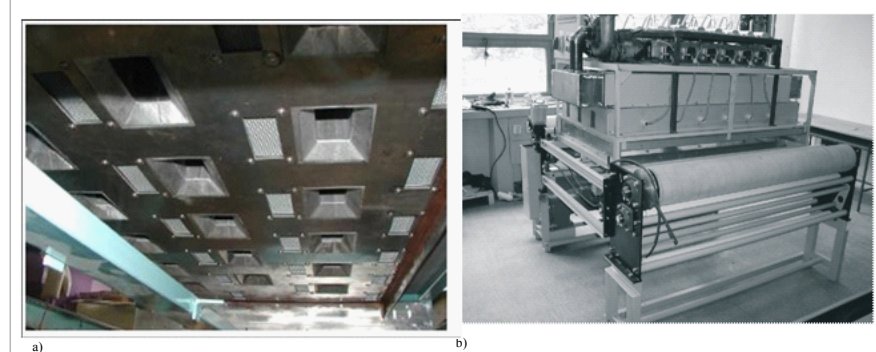

Figure 3: Open-resonator a) Interior the microwave drying machine, b) Prototype of semi-industrial microwave drying machine.

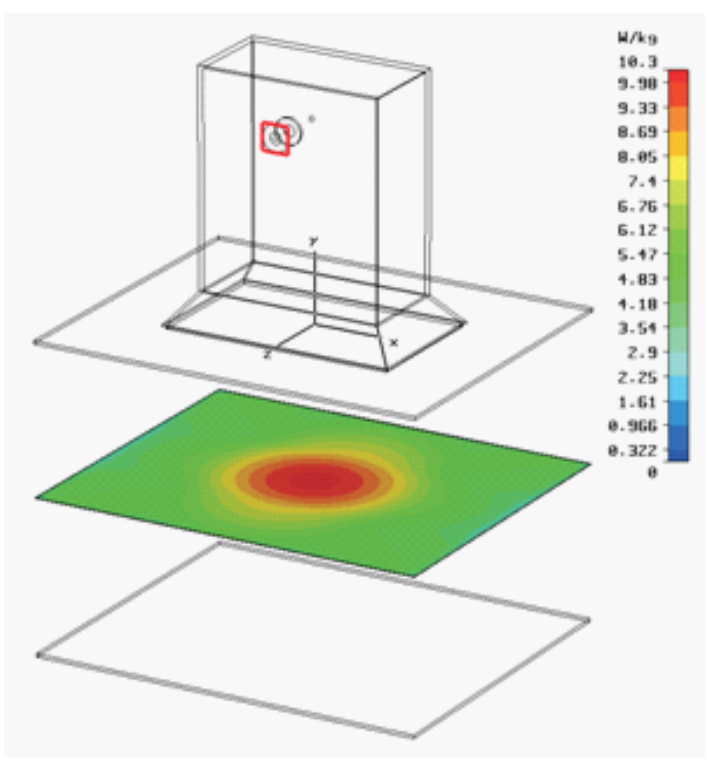

Figure 4: Distribution of electric field strength in one applicator.

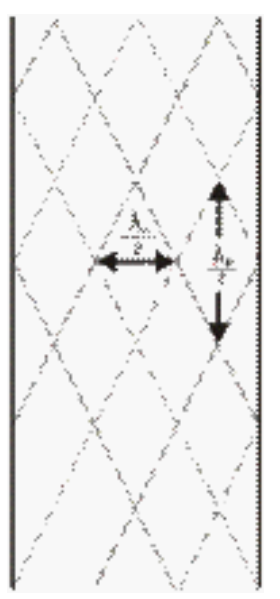

Figure 5: Characteristic wave length in waveguide.

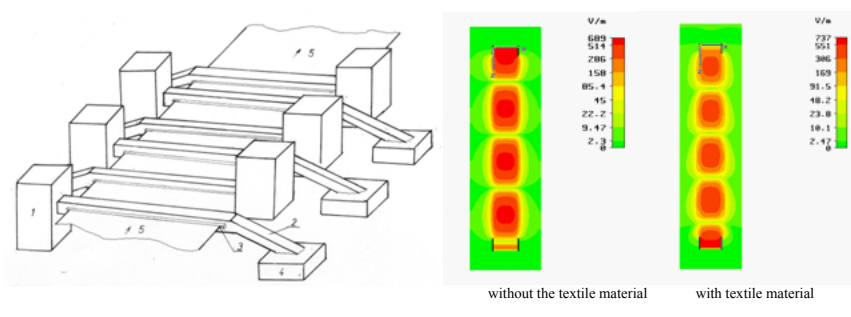

Figure 6: 2D distribution of electric field strength in one waveguide.

and be transformed into water vapor. A wet fabric submitted to a radiofrequency fields absorbs the electromagnetic energy, so that its internal temperature increases. If a sufficient amount of energy is supplied, the water is converted into steam, which leaves the product; that is to say, the wet product is dried. Radiofrequency dyers have some specific design and construction features which allow their users to obtain the maximum benefits from the radio frequency technology in terms of quality of the dried products, reduced operating costs, flexibility and reliability. The RF power adjustment is accomplished by means of a semi-automatic circuit which controls the power supplied to the product being dried through a variable capacitor, located in the generator. The electrode is fixed or automatically positioned at pre-set heights. The range of power density for textile industry is from is 3 (nylon) to $18 \mathrm{~kW} / \mathrm{m} 2$ (cotton, viscose) of electrode surface. The only devices using electromagnetic waves that are used in textile industrial applications are radio-frequency dryers.

\section{Microwave Devices in Textile Finishing}

First ideas of MW application for textile finishing processes originated in 1970's when cellulosic fabrics were treated with Durable Press finishing agents and cured in a microwave oven. Although these first results were promising, the idea was abandoned till 1995, when Miller patented his Presset process without pre-knowledge of the earlier patent. This early garment microwave project was abandoned because the efforts to control the process failed.

There are three types of devices for microwave processing of flexible materials. The device based on the resonant cavity principle can be used on discontinuing principle. Therefore it is suitable for lab 
research of small quantities of textile materials. The major part of the research was conducted on this type of a device. Devices based on the open resonator and waveguide applicator principle operate according to a continuing principle, and they are still being tested. These devices for microwave textile finishing are prevalently laboratory apparatus. Their main problem is reduced spreading of microwaves into the environment through gaps for flexible material.

\section{Resonant cavity}

The resonant cavity chamber itself is a Faraday cage which prevents the microwaves from escaping. Magnetron produce microwaves, which waveguide guiding in resonant cavity chamber. The oven door usually has a window for easy viewing, but the window has a layer of conductive mesh some distance from the outer panel to maintain the shielding. Because the size of the perforations in the mesh are much less than the microwaves' wavelength, most of the microwave radiation cannot pass through the door, while visible light (with a much shorter wavelength) can. Main problems related to the use of such resonant cavities are the non-uniform energy distribution and possible MW leakage from the door seals in the case of inadequate chokes. The distribution of microwave energy within the cavity is always imperfect and the rotator (turntable) will cause the passage of the material through hotter and cooler spots, averaging out the exposure to microwaves.

\section{Open-resonator}

The second reported microwave drying machine consist of many drying cells positioned above the moving textile material. Each of the drying cells is based on the idea of an open resonator. These cells have their own magnetron placed in a waveguide holder. This applicator, which derives from the Fabry-Perot open resonator, has a magnetron as a source of high electromagnetic power. Dried textile material is located in the middle plane between the parallel conductive plates and the distance between these plates is equal to $3 / 2 \lambda$.

In research of the open applicator they determined the position of the magnetron. In the same manner they also found the distribution of the electric field strength in drying textile materials. This applicator has a magnetron as a source of high electromagnetic power, placed in the waveguide holder. The power of the used magnetrons is $800 \mathrm{~W}$ and its working frequency is $2.45 \mathrm{GHz}$. Their drying resonant system is optimized by criteria to create the maximum electric field strength in the plane of the drying textile.

\section{Waveguide applicator}

Waveguides are metallic tubes, in the section-plane rectangle or circle. They transport electromagnetic energy from magnetron that runs along the waveguide. Waveguides work according to the principle of waves reflecting from the waveguide from one part to another. Fields in the waveguide can bee seen as a group of planar waves. They reflect from one part to another part, distributing in the direction of waveguide shown in the Figure 5.

There are two characteristic wave lengths:

- one in the direction of vertical with the waveguide:

$\lambda_{\mathrm{n}}=\lambda / \cos \theta$

- one in the direction of parallel with the waveguide: $\lambda_{\mathrm{p}}=\lambda / \sin \theta$

Were $\lambda$ is wave length appointed signal;

$\lambda_{\mathrm{n}-}$ is wave length in the direction vertical with the waveguide;

$\lambda_{p}$ I wavelength in the direction parallel with waveguide ;

$\theta$ is entrance angle (angle of incidence).

Newly designed microwave device completely utilizes the obtained microwave energy in a process. The remaining energy, not used in drying via waveguides, is used for heating the water used as the absorber of microwave energy. This heated water can be used in further processing which enables utilization of the transmitted microwave energy.

Damp textile material (5) first passes through the slots (3) in the waveguide (2). Redundant energy is guided through the waveguide to the absorber of microwave energy (water) (4). In order to obtain uniform absorption of microwave energy on the whole material, an even number of waveguides must always be used (Figure 6). The number of waveguides used depends on the desired speed of the textile material passing and the amount of water on the material.

\section{Microwave in Textile Finishing}

Possibility of microwave treatment application for different finishing processes was determined. From the results of wrinkle recovery angel (WRA) noticeable improvements can be seen in a case of treatment with microwaves. At the same time tensile strength retention has been improved, meaning that there is no usual negative interference with mechanical strength. Under the influence of microwaves formaldehyde release has been reduced by $50 \%$ compared to conventional thermal treatment what certainly gives an advantage to this method to treatment.

The results of water repellent finishing are noticeable in a case of microwave treatment. But, oil repellent finishing obtained only with additional thermal treatment, which is included in the passage of the textile material for additional improvement of fluorocarbon orientation. For good oil-repellency effects the orientation of hydrophobic atoms is primary concern. Effectives of the repellency after the dry-cleaning is very good with both applied curing methods.

Results of flame retardancy, obtained by microwave inducement, are equally good compared with the conventional curing method.

The influence of different drying methods (convection, contact and microwave), on mechanical parameters of yarn sizing has been investigated. The greatest benefit of microwave thermal treatment is in its economical efficacy, so as in uniform drying at the whole treated volume. The sizing effects obtained by microwaves are similar to the one obtained with the most often explored contact drying.

\section{Conclusion}

Today we have become surrounded by microwave radiation of various systems, from natural sources to those caused by human action. Environmental exposure to man-made electromagnetic fields has been steadily increasing due to growing electricity demand, ever-advancing technologies and changes in social behavior.

The main advantage of the microwave energy application is that the 
energy consumption is $60-70 \%$ lower respect to conventional heating treatments. Another advantage is its influence on the reaction kinetics: a reaction that takes place in two days under conventional treatment methods terminates after a few minutes applying MW energy.

The main disadvantage of a wide application of microwave energy in textile finishing is the negative influence of electromagnetic irradiation on the environment. It means that preventive security measures are needed to be developed prior to microwave energy use on a larger scale.
The exposure to an excessive level of radiation can produce hazards. The microwave radiation is non-ionizing, its main effect being of a thermal nature, commonly used in applications. The body absorbs radiation and automatically adapts to the resulting temperature increase, excess heat being removed by the blood flow. However, should the radiation become too intense, the thermal balance no longer could be restored by the body processes, and burns would occur? As microwaves tend to heat deeply into the body, one might fear deep burns would occur while the surface temperature remained acceptable. 